ARTICLE

https://doi.org/10.1038/s41467-019-12751-z

\title{
Hydrogen bonding structure of confined water templated by a metal-organic framework with open metal sites
}

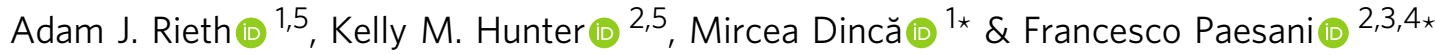

Water in confinement exhibits properties significantly different from bulk water due to frustration in the hydrogen-bond network induced by interactions with the substrate. Here, we combine infrared spectroscopy and many-body molecular dynamics simulations to probe the structure and dynamics of confined water as a function of relative humidity within a metal-organic framework containing cylindrical pores lined with ordered cobalt open coordination sites. Building upon the agreement between experimental and theoretical spectra, we demonstrate that water at low relative humidity binds initially to open metal sites and subsequently forms disconnected one-dimensional chains of hydrogen-bonded water molecules bridging between cobalt atoms. With increasing relative humidity, these water chains nucleate pore filling, and water molecules occupy the entire pore interior before the relative humidity reaches $30 \%$. Systematic analysis of rotational and translational dynamics indicates heterogeneity in this pore-confined water, with water molecules displaying variable mobility as a function of distance from the interface.

\footnotetext{
${ }^{1}$ Department of Chemistry, Massachusetts Institute of Technology, 77 Mass. Ave., Cambridge, MA 02139, USA. ${ }^{2}$ Department of Chemistry and Biochemistry, University of California San Diego, La Jolla, CA 92093, USA. ${ }^{3}$ Materials Science and Engineering, University of California San Diego, La Jolla, CA 92093, USA. ${ }^{4}$ San Diego Supercomputer Center, University of California San Diego, La Jolla, CA 92093, USA. ${ }^{5}$ These authors contributed equally: Adam J. Rieth, Kelly M. Hunter. *email: mdinca@mit.edu; fpaesani@ucsd.edu
} 


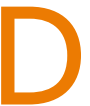
ue to the formation of frustrated hydrogen-bond (H-bond) networks, water confined within pores or at interfaces exhibits significantly altered physical properties compared to bulk water, with important implications for different fields, including chemistry ${ }^{1,2}$, biology ${ }^{3-5}$, and atmospheric science ${ }^{6,7}$. As a consequence of the unique structural and dynamical properties of frustrated H-bond networks, confinement of water gives rise to anomalous behavior, as inferred from measurements of various quantities, such as the dielectric constant ${ }^{8}$ and diffusion coefficient $^{9}$. Significant progress has recently been made in developing a more complete picture of the water $\mathrm{H}$-bonding structure ${ }^{10}$, especially due to the introduction of accurate many-body molecular models ${ }^{11}$. Nevertheless, a precise prediction of the properties of water across different phases and in different environments remains a challenge due to the dynamic nature of the $\mathrm{H}$-bond network which results from the subtle balance between energetic, entropic, and nuclear quantum effects ${ }^{11-13}$.

As confinement increases, so too does the importance of interactions between the water molecules and the confining environment, with distinct consequences for the H-bonding structure ${ }^{14,15}$. Studies aiming to characterize the thermodynamic and dynamic properties of water in confinement have targeted various porous materials, such as hydrophobic carbon nanotubes ${ }^{16-20}$ and hydrophilic zeolites and silicas, as water containers ${ }^{21}$. It was found that water inside carbon nanotubes forms one-dimensional and tightly $\mathrm{H}$-bonded chains, while ordered phases were identified in water confined in zeolites. On the other hand, water adsorbed on metal surfaces often displays well-defined patterns that are templated by the strength and anisotropy of the underlying water-metal interactions ${ }^{22}$. However, the uniformity of the hydrophobicity or hydrophilicity associated with these materials engenders mostly predictable water-substrate interactions that may differ significantly from those observed in heterogeneous environments, such as aquaporins and other structures found in biological systems ${ }^{3}$, where both hydrophilic and hydrophobic patches coexist and lead to a variety of competing $\mathrm{H}$-bonding domains ${ }^{1-7}$.

Metal-organic frameworks (MOFs) have recently received attention as water containers exhibiting tunable hydrophilicity of potential use in adsorption heat pumps ${ }^{23-28}$ and for atmospheric water harvesting 29,30 . In this regard, we posit that a MOF termed

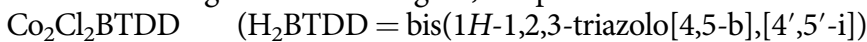
dibenzo[1,4]dioxin)), which was recently investigated for its record reversible water uptake (Fig. 1), will provide a relevant crystalline analog for investigating the $\mathrm{H}$-bonding structure of water in heterogeneous confinement ${ }^{30}$. Here we combine diffuse reflectance infrared Fourier transform spectroscopy (DRIFTS) measurements with many-body molecular dynamics (MB-MD) simulations ${ }^{31-35}$. Our analysis implicates the initial formation of distinct onedimensional (1-D) chains of adsorbed water molecules bridging between the framework hydrophilic open metal sites as the critical step for pore filling in $\mathrm{Co}_{2} \mathrm{Cl}_{2} \mathrm{BTDD}$. As the relative humidity $(\mathrm{RH})$ increases, these 1-D chains template the subsequent formation of cylindrical water shells that extend along the hydrophobic pore channels and exhibit progressively faster rotational and translational mobility as a function of the distance from the pore surface.

\section{Results}

Infrared measurements of the water adsorption process. $\mathrm{Co}_{2} \mathrm{Cl}_{2} \mathrm{BTDD}$ was synthesized as previously reported ${ }^{36}$, with a consistent powder X-ray diffraction pattern and $\mathrm{N}_{2}$ adsorption isotherm (Supplementary Figs. 6, 7). The structure exhibits hexagonal pores $2.2 \mathrm{~nm}$ in diameter linked by secondary building units consisting of infinite chains of cobalt chloride bridged by triazolate groups, wherein each nitrogen atom is ligated to a distinct $\mathrm{Co}^{2+}$ (Fig. 1a, b). DRIFTS spectra were measured at $20^{\circ} \mathrm{C}$ under variable $\mathrm{RH}$ (Fig. 2b and Supplementary Fig. 8). With increasing $\mathrm{RH}$, notable changes in the infrared spectrum appear around $600 \mathrm{~cm}^{-1}$ for the water librational mode, at $1600 \mathrm{~cm}^{-1}$ for the $\mathrm{HOH}$ bending mode, and near $3500 \mathrm{~cm}^{-1}$ for the water $\mathrm{OH}$-stretching band

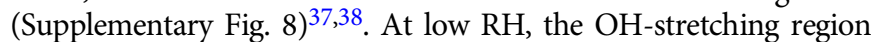
displays several well-defined peaks, indicating that the water molecules experience distinct environments within the pore, which are non-equivalent on the IR timescale (Fig. 2a). The highest frequency peak at $3700 \mathrm{~cm}^{-1}$ can be attributed to the presence of free $\mathrm{OH}$ bonds, i.e., $\mathrm{OH}$ bonds that are not engaged in $\mathrm{H}$-bonding ${ }^{39}$. Although free $\mathrm{OH}$ bonds are short-lived in bulk water ${ }^{40}$, they are present at the air/water interface ${ }^{41,42}$, which thus seems to provide a closer reference for water adsorbed at low $\mathrm{RH}$ in the $\mathrm{Co}_{2} \mathrm{Cl}_{2} \mathrm{BTDD}$ pores. The remaining series of peaks suggests a complicated interplay between water-framework and water-water interactions that lead to a broad range of $\mathrm{H}$-bond strengths.

As the RH increases, the individual peaks coalesce into a single broad band resembling that of bulk water.
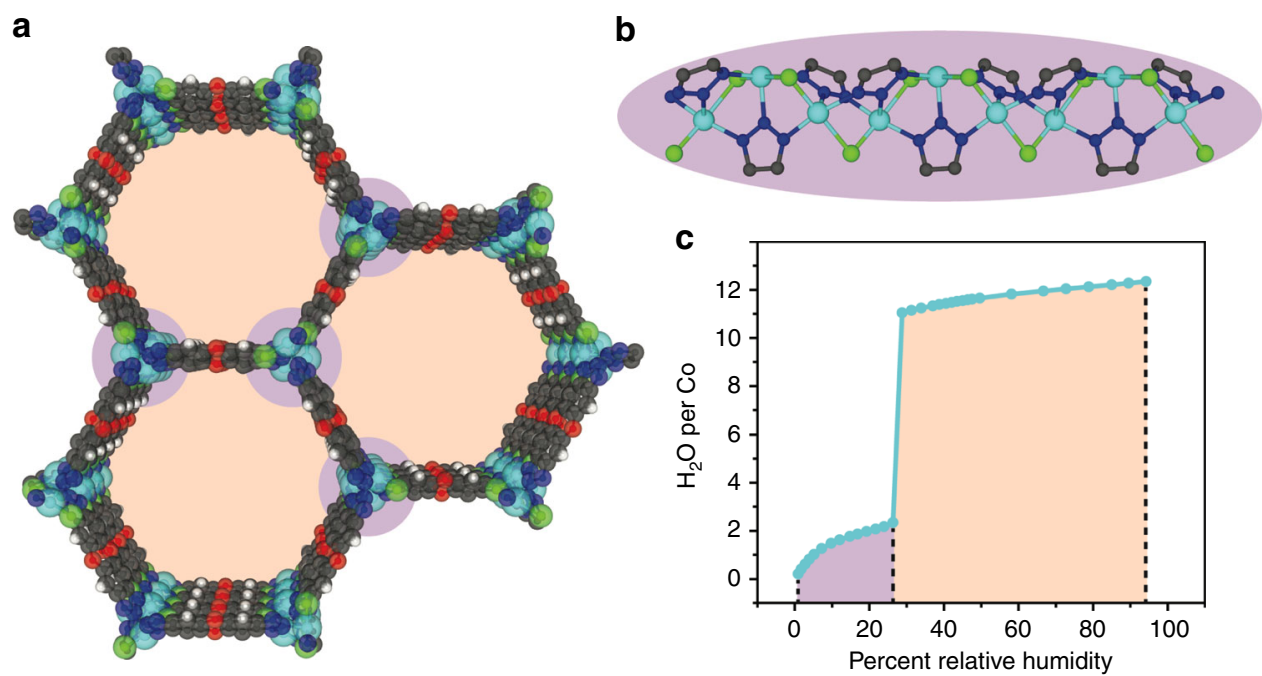

Fig. 1 Structure and Water Adsorption of $\mathrm{CO}_{2} \mathrm{Cl}$ BTDD. a Structure of $\mathrm{Co}_{2} \mathrm{Cl}_{2} \mathrm{BTDD}$ viewed down the $z$-axis. $\mathrm{C}-\mathrm{gray}, \mathrm{H}-$ white, $\mathrm{O}$ - red, $\mathrm{N}-$ dark blue, $\mathrm{Cl}$ green, $\mathrm{Co}$ - light blue. Hydrophilic regions are indicated in purple and hydrophobic regions in orange. $\mathbf{b}$ Structure of the secondary building unit along the $z$-axis. c Water adsorption isotherm at $20^{\circ} \mathrm{C}$ for $\mathrm{Co}_{2} \mathrm{Cl}_{2} \mathrm{BTDD}$ exhibiting complete pore hydration at $28 \% \mathrm{RH}$, data adapted from ref. 30 


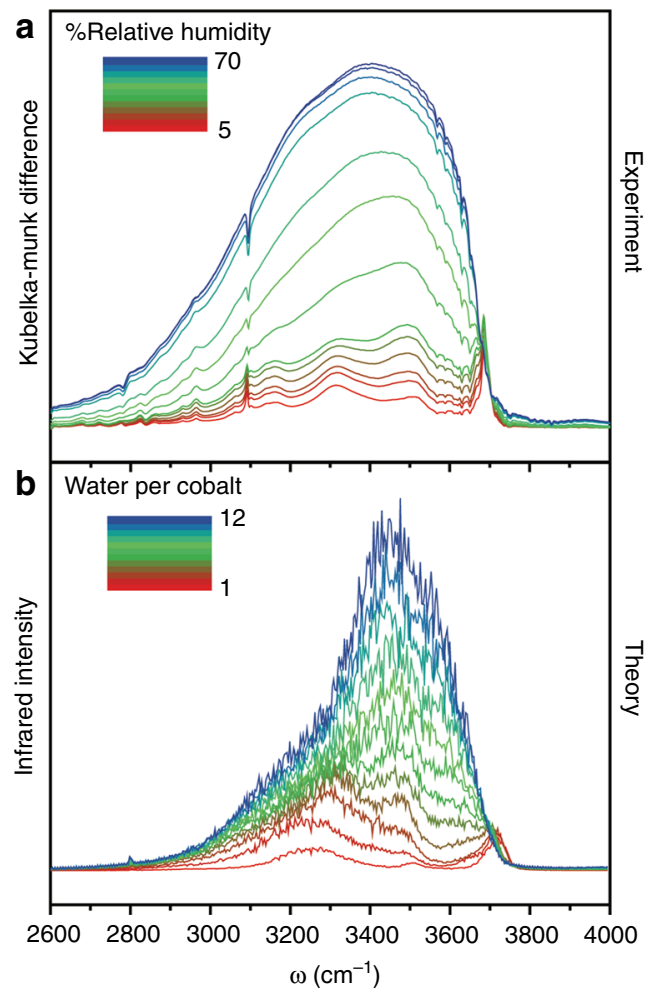

Fig. 2 Experimental and Theoretical Infrared Spectra of Water in $\mathrm{Co}_{2} \mathrm{Cl}_{2} \mathrm{BTDD}$. a Difference Diffuse-Reflectance IR spectra of the water $\mathrm{OH}$ stretch region in $\mathrm{CO}_{2} \mathrm{Cl}_{2} \mathrm{BTDD}$ under variable $\mathrm{RH}$ conditions. $\mathbf{b}$ Calculated IR intensity using the $\mathrm{MB}$-pol model of water in $\mathrm{CO}_{2} \mathrm{Cl}_{2} \mathrm{BTDD}$ ranging from one water molecule per cobalt (1) to twelve water molecules per cobalt (12)

Simulating water in confinement using the MB-pol model. A molecular-level interpretation of the origin and evolution of the different spectral features as a function of $\mathrm{RH}$ is obtained from classical MB-MD simulations carried out combining the MB-pol water model ${ }^{31-35}$, which has been shown to accurately predict the properties of water from the gas to the condensed phase ${ }^{43}$, with a flexible force field for $\mathrm{Co}_{2} \mathrm{Cl}_{2} \mathrm{BTDD}$ (see the Supplementary Methods, Supplementary Figs. 1-5, and Supplementary Tables 14 for details). All simulations were carried out at a temperature of $300 \mathrm{~K}$ (Supplementary Figs. 14, 15). To allow for direct comparison with the experimental data, all theoretical infrared (IR) spectra, which are calculated from the dipole-dipole time correlation function (see the Supplementary Methods for details), are red-shifted by $175 \mathrm{~cm}^{-1}$ in the $\mathrm{OH}$-stretching region to account for zero-point energy effects that are neglected in classical MBMD simulations, as discussed in detail in Refs. ${ }^{34,44}$. Accounting for zero-point energy effects, good agreement is obtained with the experimental data (Fig. 2b and Supplementary Fig. 8), with the theoretical spectra reproducing the same distinct series of peaks at low $\mathrm{RH}$ and the emergence of a progressively broader band as the $\mathrm{RH}$ increases.

\section{Discussion}

To characterize the evolution of the H-bond network during pore filling, the results of separate MB-MD simulations carried out with only one and two water molecules in the simulation box are examined in Fig. 3a. The most favorable location of a single water molecule in the $\mathrm{Co}_{2} \mathrm{Cl}_{2} \mathrm{BTDD}$ pores corresponds to coordination with one of the open cobalt sites. In this configuration, the two $\mathrm{OH}$ bonds of the water molecule are not equivalent on a picosecond timescale due to different interactions with the
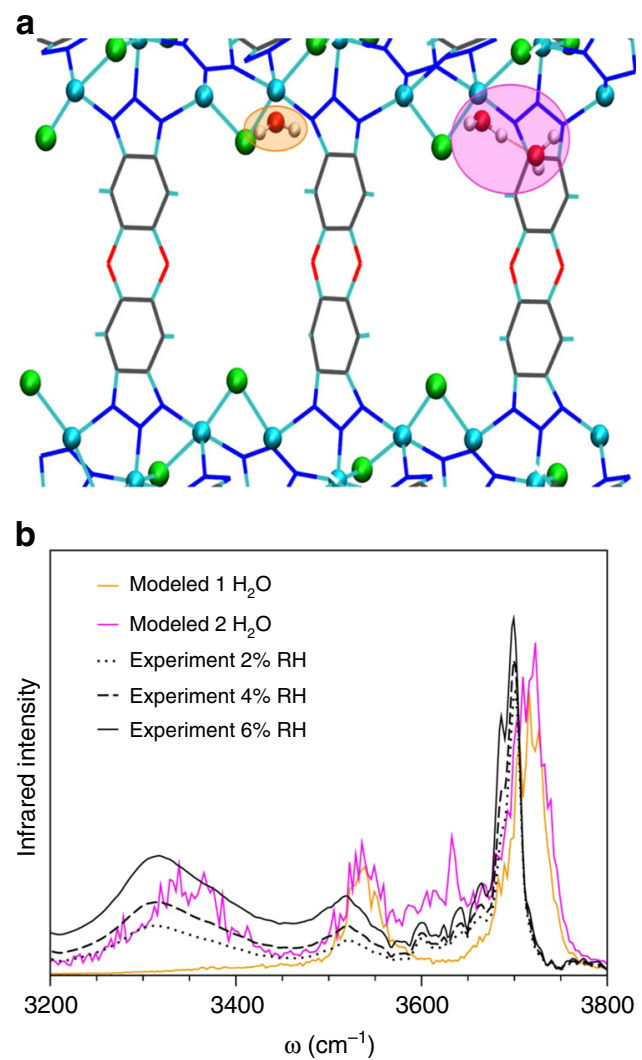

Fig. 3 Water at Low Loadings in $\mathrm{Co}_{2} \mathrm{Cl}_{2} \mathrm{BTDD}$. a Simulations with one (orange) and two (pink) water molecules. Distance shown along the $z$-axis is $24 \AA$. b Calculated IR OH-stretches of one (orange) and two (pink) water molecules with corresponding experimental DRIFTS spectra at RH $2 \%$ (dotted black), 4\% (dashed black), and 6\% (solid black)

framework. This results in two peaks at $3550 \mathrm{~cm}^{-1}$ and $3700 \mathrm{~cm}^{-1}$ (orange trace in Fig. 3b) that, while reminiscent of the symmetric and asymmetric stretches, correspond to two distinct $\mathrm{OH}$-stretch vibrations (see Supplementary Fig. 9 for specific details, and Supplementary Tables 6-17 and Supplementary Figs. 14, 15 for details concerning simulation temperature at various water loadings). The peak at lower frequency is associated with the stretching vibration of the $\mathrm{OH}$ bond weakly interacting with a triazolate group of the framework while the peak at higher frequency is associated with the $\mathrm{OH}$ bond weakly interacting with the nearest chloride atom of the framework.

The addition of a second water molecule leads to the formation of a H-bonded dimer, with the first molecule remaining coordinated with the open cobalt site. MB-MD simulations indicate that the two $\mathrm{OH}$ bonds on the second water are relatively free to rotate (on a timescale of $\sim 0.50 \mathrm{ps}$ ) due to lack of specific interactions with the framework. The decomposition of the theoretical IR spectrum in terms of individual $\mathrm{OH}$-stretch contributions (pink trace in Fig. $3 \mathrm{~b}$ ) reveals that the second water molecule is primarily responsible for the peaks at $3550 \mathrm{~cm}^{-1}$ and $3700 \mathrm{~cm}^{-1}$. The cobalt-bound water is instead responsible for the emergence of the two new peaks at $3300 \mathrm{~cm}^{-1}$ and $3650 \mathrm{~cm}^{-1}$, with the first peak corresponding to the $\mathrm{H}$-bonded $\mathrm{OH}$-stretch and the second peak being associated with the non- $\mathrm{H}-$-bonded $\mathrm{OH}$ bond. Being relatively free to rotate, the non- $\mathrm{H}$-bonded $\mathrm{OH}$ bond experiences a wide range of local environments and is thus found to also contribute to the peaks at $3550 \mathrm{~cm}^{-1}$ and $3700 \mathrm{~cm}^{-1}$ (see Supplementary Fig. 9 for specific details).

A nearly one-to-one correspondence is found in Fig. 3b between the theoretical IR spectra calculated for one and two 

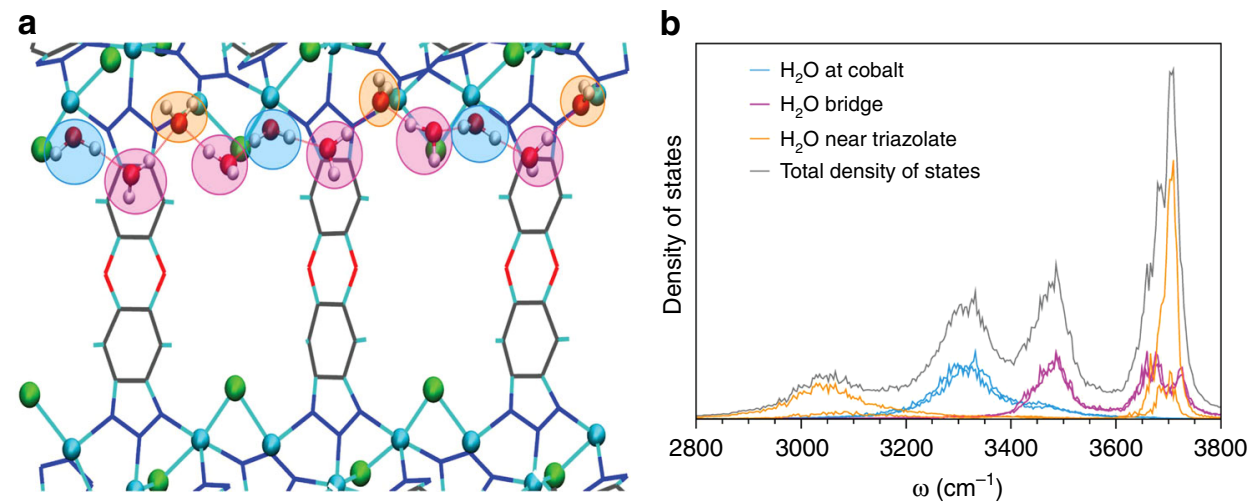

Fig. 4 Chains of Water Bridge Between Cobalt Sites. a Structure of the one-dimensional water chain with water at cobalt (blue), water bridges (pink), and water near the triazolate (orange) highlighted. Distance shown along the $z$-axis is $24 \AA$. b Density of states calculated for individual hydrogen atoms

water molecules and the experimental DRIFTS spectra measured at low $\mathrm{RH}$. Based on the equilibrium water isotherm data (Fig. 1c), the experimental water uptake at 2, 4, and $6 \% \mathrm{RH}$ corresponds to approximately $0.5,0.9$, and 1.2 water molecules per cobalt atom, respectively. Although water molecules interact more strongly with the open $\mathrm{Co}^{2+}$ sites, the presence of the spectral feature at $3300 \mathrm{~cm}^{-1}$, indicative of the water dimer at the cobalt site, thus implies that water begins forming localized $\mathrm{H}$ bonded clusters seeded by cobalt-bound water molecules prior to full saturation of the open $\mathrm{Co}^{2+}$ sites.

Simulating the adsorption of additional water molecules reveals the formation of 1-D water chains bridging between cobalt sites. These chains consist of three types of water molecules residing in three distinct local environments. Water molecules of the first type correspond to those bound to the open $\mathrm{Co}^{2+}$ sites (light blue in Fig. 4a), which engage in two H-bonds, one each to two adjacent water molecules of the second type. These second-type water molecules (pink in Fig. 4a) act as bridging molecules (similar to the second water molecule in Fig. 3a) by accepting and donating one $\mathrm{H}$-bond and do not interact directly with the framework. Water molecules of the third type (orange in Fig. 4a) correspond to those that interact with the framework by pointing one $\mathrm{OH}$ bond to the $\pi$-system of the neighboring triazolate group, while accepting two $\mathrm{H}$-bonds, one each from two adjacent bridging water molecules.

Further insights into the nature of the interactions between the framework and the water molecules residing in the three different local environments are gained by dissecting the density of states (DOS), corresponding to the theoretical spectra, into individual contributions associated with each type of water molecule along the 1-D chains. It is found that the cobalt-bound water molecules only contribute to the peak at $3300 \mathrm{~cm}^{-1}$ (light blue trace in Fig. 4b), which is a spectral feature characteristic of double donor $\mathrm{H}$-bonded water molecules. Since these molecules are pinned to the open $\mathrm{Co}^{2+}$ sites and engage in two $\mathrm{H}$-bonds, which are equivalent on the IR timescale, their mobility is highly frustrated (Supplementary Fig. 10). The water-bridging molecules contribute to the two peaks at 3500 and $3700 \mathrm{~cm}^{-1}$ (pink trace in Fig. $4 \mathrm{~b}$ ), which are associated with the stretching vibrations of the $\mathrm{H}$-bonded and free $\mathrm{OH}$ bonds, respectively. Due to the absence of directional interactions with the framework, these molecules can easily switch $\mathrm{H}$-bond partners and display significantly faster orientational mobility than the cobalt-bound molecules (Supplementary Fig. 10). Finally, the water-to-triazolate molecules contribute to the two bands at $\sim 3050$ and $3700 \mathrm{~cm}^{-1}$ (orange trace in Fig. $4 \mathrm{~b}$ ), with the lower frequency peak being associated with the stretching vibrations of the $\mathrm{OH}$ bonds pointing toward the $\pi$-systems of the triazolate groups and the higher frequency peak corresponding to the stretching vibrations of the other (free) $\mathrm{OH}$ bonds, respectively. Due to relatively stronger interactions with the triazolate groups, these water molecules exhibit rotational mobility that is intermediate between those displayed by cobaltbound and bridging water molecules (Supplementary Fig. 10). The combination of the theoretical DOSs calculated for the three types of water molecules along the 1-D chains results in a vibrational lineshape (gray trace in Fig. 4b) that reproduces the main peaks of the experimental DRIFTS spectra measured below $30 \%$ RH, before the MOF pores become fully hydrated (Fig. 2a and Supplementary Table 5). Although connecting every open $\mathrm{Co}^{2+}$ site with water chains would require four water molecules per cobalt, the isotherm data indicate that pore hydration is initiated at 2.3 water molecules per cobalt (Fig. 1c). This suggests that disconnected 1-D chains form in various locations within the framework before all cobalt sites are saturated, which is supported by our MB-MD simulations showing that $1-\mathrm{D}$ chains bridging multiple cobalt sites begin to appear at a loading of two water molecules per cobalt.

At higher water loadings, the 1-D water chains bridging the hydrophilic open $\mathrm{Co}^{2+}$ sites act as nucleators for the pore filling process, templating the formation of concentric cylindrical shells that extend along the hydrophobic pore channels. As the water loading increases, the MB-MD simulations indicate that the water molecules become, on average, more mobile (Fig. 5a). Because the orientational correlation functions reflect the extent of molecular rotation over time, this suggests the emergence of liquid-like behavior. However, at the experimental maximum loading of 12 $\mathrm{H}_{2} \mathrm{O} / \mathrm{Co}^{2+}$, the average orientational mobility of the water molecules in the $\mathrm{Co}_{2} \mathrm{Cl}_{2} \mathrm{BTDD}$ pores remains intermediate between that calculated for ice and bulk water. A similar slowdown was predicted for water adsorbed in MIL-53 ${ }^{45}$.

The evolution of the dynamical behavior of water adsorbed in $\mathrm{Co}_{2} \mathrm{Cl}_{2} \mathrm{BTDD}$ as a function of $\mathrm{RH}$ can be further characterized by analyzing the variation of the water mobility from the surface to the center of the pore. To this purpose, the water molecules along the MB-MD trajectories are classified based on their distances from the surface of the pore and thus assigned to three concentric cylindrical sectors (shown in dark yellow, orange, and red in Fig. 5b, e), with a width of $4.0 \AA$ each.

As discussed above, at low $\mathrm{RH}$ corresponding to a loading of 1 $\mathrm{H}_{2} \mathrm{O} / \mathrm{Co}^{2+}$, the water molecules are primarily coordinated to the open cobalt sites, although there is not a one-to-one correspondence, and $\mathrm{Co}^{2+}$-pinned water dimers and trimers also form within the pores as inferred by the DRIFTS lineshapes. These water molecules thus reside in sector 1 , providing an outer shell of $\mathrm{H}$-bonding sites that effectively template the development of the $\mathrm{H}$-bonding structure inside the pores at high $\mathrm{RH}$ (Fig. 5b, c). Water 
a

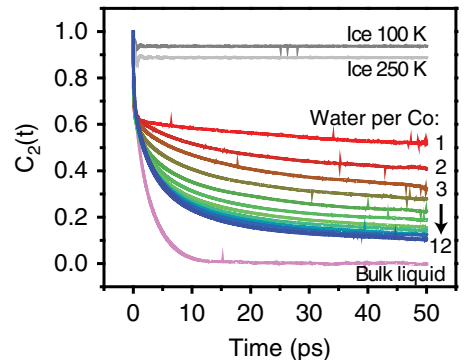

d

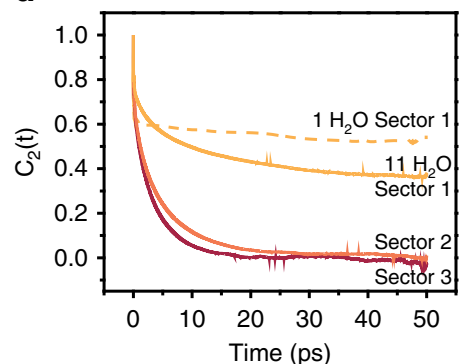

b

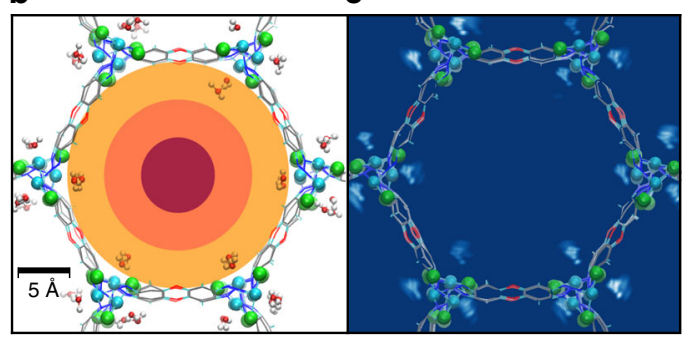

e

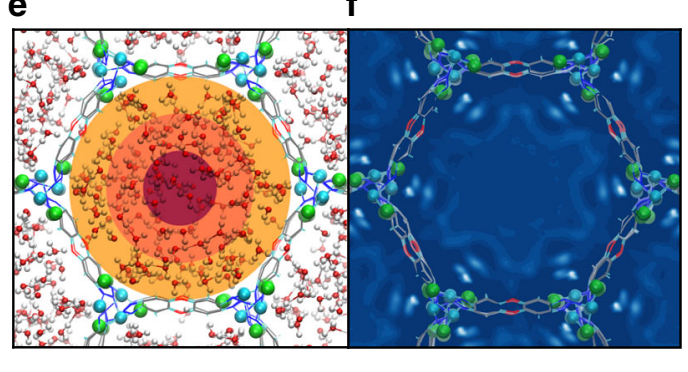

Fig. 5 Dynamics of Water in $\mathrm{CO}_{2} \mathrm{Cl}_{2} \mathrm{BTDD}$. a Orientational correlation functions calculated from MB-MD simulations carried out for various loadings of water inside the $\mathrm{CO}_{2} \mathrm{Cl}_{2} \mathrm{BTDD}$ pores. Also shown for reference are the corresponding orientational correlation functions calculated from $\mathrm{MB}-\mathrm{MD}$ simulations of liquid water (pink) and ice (gray). b Snapshot from an MB-MD simulation of water in $\mathrm{Co}_{2} \mathrm{Cl}_{2} \mathrm{BTDD}_{\mathrm{T}}$ for a loading of $1 \mathrm{H}{ }_{2} \mathrm{O} / \mathrm{Co}{ }^{2+}$. The three concentric colored sectors are defined according to their distance from the pore surface: Sector 1 from 0-4 $\AA$ (dark yellow), Sector 2 from $4-8 \AA$ (orange), and Sector 3 from 8-12 A (red). c Two-dimensional (2-D) density map of water calculated from MB-MD simulations carried out for a loading of $1 \mathrm{H}_{2} \mathrm{O} / \mathrm{Co}{ }^{2+}$ (lighter colors correspond to regions with higher water density). d Orientational correlation functions calculated for water in the three different sectors of the pore at either $1 \mathrm{H}_{2} \mathrm{O} / \mathrm{Co}^{2+}$ (dashed line) or $11 \mathrm{H}_{2} \mathrm{O} / \mathrm{Co}^{2+}$ (solid lines). e Snapshot of an $\mathrm{MB}-\mathrm{MD}$ simulation of water in $\mathrm{Co}_{2} \mathrm{Cl}_{2} \mathrm{BTDD}^{2}$ for a loading of $11 \mathrm{H}_{2} \mathrm{O} / \mathrm{Co}^{2+}$ (the three concentric colored sectors are defined as in $\mathbf{b}$. $\mathbf{f} 2-\mathrm{D}$ density map for a loading of $11 \mathrm{H}_{2} \mathrm{O} / \mathrm{Co}^{2+}$ (lighter colors correspond to regions with higher water density)

molecules enter sector 2 at a loading of $2 \mathrm{H}_{2} \mathrm{O} / \mathrm{Co}^{2+}$ and start filling sector 3 at a loading of $7 \mathrm{H}_{2} \mathrm{O} / \mathrm{Co}^{2+}$ (Fig. $5 \mathrm{~d}$ and Supplementary Figs. 11, 12). At the loading of $11 \mathrm{H}_{2} \mathrm{O} / \mathrm{Co}^{2+}$, the water molecules fill the pore completely although they tend to cluster around the open cobalt sites as shown in Fig. 5e, f. Similar templating effects of the framework on adsorbed water were predicted from computer simulations of water in $[\mathrm{Zn}(\mathrm{L})(\mathrm{X})](\mathrm{L}=3$-methyl-2-(pyridin-4ylmethylamino)-butanoic acid and $\mathrm{X}=\mathrm{Cl}$ and $\mathrm{Br})^{46}$, and MOF$74^{47}$. Analysis of both orientational and translational mobility shows that water molecules occupying different sectors exhibit significantly different dynamical behavior (Table 1 and Supplementary Figs. 11, 12). While water molecules in sector 1 reorient very slowly and are effectively translationally immobile, being directly coordinated with the $\mathrm{Co}^{2+}$ atoms or H-bonded to the Cobound molecules, water molecules in sectors 2 and 3 display progressively faster dynamics. In particular, water molecules at the center of the pore (sector 3 ) reorient on a timescale of $\sim 4.4 \mathrm{ps}$ and diffuse along the pore ( $z$ direction) by $0.24 \AA^{2} \mathrm{ps}^{-1}$, which suggests dynamical behavior similar to liquid water (Table 1 ). The overall difference in the orientational relaxation times and diffusion coefficients of Table 1 displays the dynamical heterogeneity that exists between the different sectors due to their distance from the pore surface. Additionally, it should be noted that, due to confinement, water mobility along the $x$ and $y$ directions is systematically lower than along the $z$ direction.

In addition to the dynamical heterogeneity exhibited by water molecules in different pore environments, structural parameters reveal increasingly frustrated $\mathrm{H}$-bond networks closer to the pore surface. At the maximum loading of $12 \mathrm{H}_{2} \mathrm{O} / \mathrm{Co}^{2+}$, Supplementary Fig. 13 shows that the distribution of the tetrahedral order parameter, $\mathrm{q}_{\text {tet }}$, a metric of the local structure of the water $\mathrm{H}$ bond network, displays two distinct features-a dominant one
Table 1 Dynamics of water in $\mathrm{Co}_{2} \mathrm{Cl}_{2} \mathrm{BTDD}$ orientational relaxation time $\left(\boldsymbol{\tau}_{\mathbf{2}}\right)$, calculated from $C_{2}(t)=A e^{-t / T_{2}}$, along with the total diffusion coefficient $\left(D_{\text {tot }}\right)$, diffusion coefficient along the $x y$-plane $\left(D_{x y}\right)$, and diffusion coefficient along the $z$ direction $\left(D_{z}\right)$ for $11 \mathrm{H}_{2} \mathrm{O} / \mathrm{Co}^{2+}$ with standard deviations. Experimental data for $\tau_{2}$ and $D_{\text {tot }}$ in bulk water are taken from refs. 49,50 , respectively. See the Supplementary Methods for specific details about the calculation of orientational relaxation time and diffusion coefficients

\begin{tabular}{lllll} 
& $\boldsymbol{\tau}_{\mathbf{2}}(\mathbf{p s})$ & $\mathbf{D}_{\text {tot }}\left(\AA^{\mathbf{2}} \cdot \mathbf{p s}^{-\mathbf{1})}\right.$ & $\mathbf{D}_{\mathbf{x y}}\left(\AA^{\mathbf{2}} \cdot \mathbf{p s}^{-\mathbf{1}}\right)$ & $\mathbf{D}_{\mathbf{z}}\left(\AA^{\mathbf{2}} \cdot \mathbf{p s}^{-\mathbf{1}}\right)$ \\
\hline Sector 1 & $>70$ & $0.03 \pm 0.01$ & $0.02 \pm 0.01$ & $0.04 \pm 0.01$ \\
Sector 2 & $8.3 \pm 1.8$ & $0.11 \pm 0.03$ & $0.09 \pm 0.03$ & $0.15 \pm 0.04$ \\
Sector 3 & $4.4 \pm 0.9$ & $0.19 \pm 0.05$ & $0.16 \pm 0.05$ & $0.24 \pm 0.08$ \\
Exp. Bulk water & 2.5 & 0.23 & &
\end{tabular}

No standard deviations were calculated for the orientational relaxation time in Sector 1 since it was only possible to determine that the relaxation time is longer than $70 \mathrm{ps}$

with a maximum at $\mathrm{q}_{\text {tet }} \approx 0.4$, indicating less tetrahedrality and suggesting environments with interfacial character, and a second feature with a maximum at $\mathrm{q}_{\mathrm{tet}} \approx 0.8$, representative of liquid-like environments ${ }^{48}$. The structural and dynamical heterogeneity exhibited by water at the maximum loading thus mirrors the water adsorption process observed by DRIFTS, as the sites with the highest water density, the least tetrahedral H-bonding network, and the slowest mobility in the full pore correspond with the sites of initial water adsorption near the metal sites.

Water confined in $\mathrm{Co}_{2} \mathrm{Cl}_{2} \mathrm{BTDD}$ pores exhibits similarities and differences with water adsorbed on surfaces. For example, water at metal surfaces tends to display long-range order ${ }^{51}$. Although at low 
$\mathrm{RH}$, water inside $\mathrm{Co}_{2} \mathrm{Cl}_{2} \mathrm{BTDD}$ displays a similar order due to coordination to the open cobalt sites, higher $\mathrm{RH}$ disrupts the longrange order, and water molecules display progressively liquid-like behavior as they approach the center of the MOF pores. This is reflected in the broadening of the $\mathrm{OH}$-stretch vibrational lineshapes towards lower frequencies characteristic of $\mathrm{H}$-bond networks.

In summary, water adsorbed in $\mathrm{Co}_{2} \mathrm{Cl}_{2} \mathrm{BTDD}$ displays heterogeneous structural and dynamical behavior which varies as a function of both $\mathrm{RH}$ and distance from the pore surface. By directly connecting adsorption isotherms with the evolution of IR spectra of water inside MOF pores as a function of $\mathrm{RH}$, the foregoing combined experimental and theoretical approach provides detailed insights into the molecular mechanisms that determine water adsorption in porous materials exhibiting both hydrophilic and hydrophobic regions. These mechanistic insights can contribute to the design of next-generation porous materials for water harvesting. Fundamentally, our approach advances the understanding of water structure and dynamics within amphipathic confined and interfacial environments which are widespread in biology, atmospheric science, and chemistry.

\section{Methods}

Synthesis. $\mathrm{Co}_{2} \mathrm{Cl}_{2} \mathrm{BTDD}$ was synthesized and activated according to a previously published procedure ${ }^{36}$. Briefly, $200 \mathrm{mg} \mathrm{H}_{2} \mathrm{BTDD}^{3}(0.75 \mathrm{mmol})$ was dissolved in $200 \mathrm{~mL}$ N,N'-dimethylformamide (DMF) with heating, then cooled to room temperature. Separately, $1.5 \mathrm{mmol}$ ( 2 eq.) cobalt chloride hydrate was dissolved in $200 \mathrm{~mL}$ ethanol and $4 \mathrm{~mL}$ concentrated hydrochloric acid. The clear solutions were combined, capped, and heated to $65^{\circ} \mathrm{C}$ in an oven for 10 days. The resulting solids were filtered, washed with DMF and methanol. Solvent exchange of DMF was carried out by Soxhlet extraction with methanol for approximately $48 \mathrm{~h}$. The materials were then activated under dynamic vacuum at $150^{\circ} \mathrm{C}$ for $24 \mathrm{~h}$.

\section{Spectroscopic measurements. Diffuse reflectance infrared Fourier transform} spectroscopy (DRIFTS) measurements were performed using a Bruker Tensor 37 IR spectrometer equipped with a liquid nitrogen cooled mercury cadmium telluride detector and a Pike DiffusIR accessory. A sample of $\mathrm{Co}_{2} \mathrm{Cl}_{2} \mathrm{BTDD}$, pre-activated at $150{ }^{\circ} \mathrm{C}$ under vacuum to remove all solvents, was diluted with $\mathrm{KBr}$ in a ratio of approximately 1:5 (MOF:KBr) in an argon-filled glovebox. The resulting solid solution was then packed into a ceramic cup and sealed in the DiffusIR cell. The cell was brought out of the box, and a static dry spectrum was recorded with the cell sealed. Two gas streams of flowing argon (UHP grade 5.0, Airgas), one wet (bubbled through a fine frit through MilliQ $\mathrm{H}_{2} \mathrm{O}$ ) and one dry, were each flow controlled using mass flow controllers (MFCs), and joined together at a $\mathrm{T}$ fitting before connecting to the DRIFTS cell. The wet stream and dry stream were controlled via the MFCs to change relative humidity (RH) every $20 \mathrm{~min}$ (a time period previously demonstrated to result in saturation of the IR spectrum at all loadings). The MFCs were controlled such that the total flow rate was constant at 1 liter per minute (LPM) (e.g. for $40 \% \mathrm{RH}, 0.4 \mathrm{LPM}$ wet, $0.6 \mathrm{LPM}$ dry). The temperature for all measurements was $20^{\circ} \mathrm{C}$. Spectra were recorded at the end of the period at which the sample atmosphere was at each RH, every $20 \mathrm{~min}$. Data was transformed using the Kubelka-Munk function ${ }^{52}$. The static dry spectrum was subtracted from the humid measurements in all cases.

Molecular dynamics simulations. All simulations utilized the many-body potential energy function (MB-pol) to describe water, which is built upon a manybody expansion of the interaction energy for water ${ }^{31-33}$. MB-pol has been previously shown to accurately reproduce the properties of water from the gas to the condensed phase ${ }^{35}$. The framework atoms of $\mathrm{Co}_{2} \mathrm{Cl}_{2} \mathrm{BTDD}$ were modeled with a flexible force field consisting of point charges (details in the Supplementary Methods). In simulating $\mathrm{Co}_{2} \mathrm{Cl}_{2} \mathrm{BTDD}$, the first configuration utilizes one water molecule per cobalt atom (1, corresponding to 54 water molecules), and in each subsequent simulation at each loading, an additional water molecule is added per cobalt atom $(2,3$, etc.). The initial configurations for each loading were generated using Packmol ${ }^{53}$, adding the specific number of water molecules to $756 \mathrm{MOF}$ atoms. Classical many-body molecular dynamics (MB-MD) simulations were performed using in-house software based on the DL_POLY_2 simulation package $^{54}$, which was modified to include the MB-pol potential energy function ${ }^{31-33}$ All simulations were carried out for a system consisting of $1 \times 1 \times 3$ primitive cells under periodic boundary conditions with cell dimensions $38.6590 \AA$, $33.4793 \AA$, and $25.6914 \AA$ and angles $90^{\circ}, 90^{\circ}$, and $120^{\circ}$ along the $x, y$, and $z$ dimensions, respectively. Each system was equilibrated through a constant volume and constant temperature (NVT) canonical ensemble at $300 \mathrm{~K}$ for $10 \mathrm{ps}$, and dynamical information was obtained through a constant volume and constant energy (NVE) microcanonical ensemble for 50 ps where the temperature remained stable around $300 \mathrm{~K}$. Constant pressure and constant temperature (NPT) simulations at $1.0 \mathrm{~atm}$ and $300 \mathrm{~K}$ were also performed to investigate the flexibility of the framework throughout the simulation. During the NPT simulations, all three cell dimensions vary by $\sim 4.5 \%$ with average values of $36.6955 \AA$, $31.7789 \AA$, and $24.3865 \AA$ along the $x, y$, and $z$ dimensions, respectively. While the cell size varies slightly, the framework remains constant in size throughout the simulations. Twenty independent MB-MD trajectories starting from different initial configurations were performed for each loading with a time step of $0.2 \mathrm{fs}$. The equations of motion were propagated according to the velocity-Verlet algorithm, and the temperature was maintained at $300 \mathrm{~K}$ by a Nosé-Hoover chain of four thermostat $\mathrm{s}^{55}$. Short-range interactions were truncated at an atom-atom distance of $9.0 \AA$, and the electrostatics were calculated using the Ewald sum ${ }^{56}$. Standard long-range electrostatic interactions as implemented in DL_POLY_2 were applied to Lennard-Jones potentials to account for errors due to the truncation at $9 \AA^{54}$. Cross interactions between water and the MOF were derived from Lorentz-Berthelot mixing rules.

\section{Data availability}

Any data generated and analyzed for this study that are not included in this Article and its Supplementary Information are available from the authors upon request.

\section{Code availability}

The MB-pol water model used in this study is available in OpenMM (http:// paesanigroup.ucsd.edu/software/mbpol_openmm.html) and i-PI (http://paesanigroup. ucsd.edu/software/mbx.html). All computer codes used in the analysis presented in this study are available from the authors upon request.

Received: 29 April 2019; Accepted: 26 September 2019; Published online: 18 October 2019

\section{References}

1. Davis, J. G., Gierszal, K. P., Wang, P. \& Ben-Amotz, D. Water structural transformation at molecular hydrophobic interfaces. Nature 491, 582-585 (2012).

2. Koga, K., Gao, G. T., Tanaka, H. \& Zeng, X. C. Formation of ordered ice nanotubes inside carbon nanotubes. Nature 412, 802-805 (2001).

3. Eriksson, U. K. et al. Subangstrom resolution X-ray structure details aquaporin-water interactions. Science 340, 1346-1349 (2013).

4. Fayer, M. D. \& Levinger, N. E. Analysis of water in confined geometries and at interfaces. Annu. Rev. Anal. Chem. 3, 89-107 (2010).

5. Hudait, A. et al. Preordering of water is not needed for ice recognition by hyperactive antifreeze proteins. Proc. Natl Acad. Sci. USA 115, 8266-8271 (2018).

6. Kiselev, A. et al. Active sites in heterogeneous ice nucleation-the example of K rich feldspars. Science 355, 367-371 (2017).

7. Qiu, Y. Q. et al. Ice nucleation efficiency of hydroxylated organic surfaces is controlled by their structural fluctuations and mismatch to ice. J. Am. Chem. Soc. 139, 3052 (2017).

8. Fumagalli, L. et al. Anomalously low dielectric constant of confined water. Science 360, 1339-1342 (2018).

9. Radha, B. et al. Molecular transport through capillaries made with atomicscale precision. Nature 538, 222-225 (2016).

10. Pettersson, L. G. M., Henchman, R. H. \& Nilsson, A. Water-the most anomalous liquid. Chem. Rev. 116, 7459-7462 (2016).

11. Cisneros, G. A. et al. Modeling molecular interactions in water: from pairwise to many-body potential energy functions. Chem. Rev. 116, 7501-7528 (2016).

12. Gallo, P. et al. Water: a tale of two liquids. Chem. Rev. 116, 7463-7500 (2016).

13. Ceriotti, M. et al. Nuclear quantum effects in water and aqueous systems: experiment, theory, and current challenges. Chem. Rev. 116, 7529-7550 (2016).

14. Levinger, N. E. Water in confinement. Science 298, 1722-1723 (2002).

15. Cerveny, S., Mallamace, F., Swenson, J., Vogel, M. \& Xu, L. M. Confined water as model of supercooled water. Chem. Rev. 116, 7608-7625 (2016).

16. Tunuguntla, R. H. et al. Enhanced water permeability and tunable ion selectivity in subnanometer carbon nanotube porins. Science 357, 792-796 (2017).

17. Byl, O. et al. Unusual hydrogen bonding in water-filled carbon nanotubes. J. Am. Chem. Soc. 128, 12090-12097 (2006).

18. Hummer, G., Rasaiah, J. C. \& Noworyta, J. P. Water conduction through the hydrophobic channel of a carbon nanotube. Nature 414, 188-190 (2001).

19. Vaitheeswaran, S., Rasaiah, J. C. \& Hummer, G. Electric field and temperature effects on water in the narrow nonpolar pores of carbon nanotubes. J. Chem. Phys. 121, 7955-7965 (2004).

20. Rasaiah, J. C., Garde, S. \& Hummer, G. Water in nonpolar confinement: From nanotubes to proteins and beyond. Annu. Rev. Phys. Chem. 59, 713-740 (2008). 
21. Crupi, V., Longo, F., Majolino, D. \& Venuti, V. Vibrational properties of water molecules adsorbed in different zeolitic frameworks. J. Phys.: Condens. Matt. 18, 3563 (2006).

22. Carrasco, J., Hodgson, A. \& Michaelides, A. A molecular perspective of water at metal interfaces. Nat. Mater. 11, 667-674 (2012).

23. Furukawa, H. et al. Water adsorption in porous metal-organic frameworks and related materials. J. Am. Chem. Soc. 136, 4369-4381 (2014).

24. Canivet, J., Fateeva, A., Guo, Y. M., Coasne, B. \& Farrusseng, D. Water adsorption in MOFs: fundamentals and applications. Chem. Soc. Rev. 43, 5594-5617 (2014).

25. de Lange, M. F., Verouden, K., Vlugt, T. J. H., Gascon, J. \& Kapteijn, F. Adsorption-driven heat pumps: the potential of metal-organic frameworks. Chem. Rev. 115, 12205-12250 (2015)

26. Rieth, A. J. et al. Tunable metal-organic frameworks enable high-efficiency cascaded adsorption heat pumps. J. Am. Chem. Soc. 140, 17591-17596 (2018).

27. Wade, C. R., Corrales-Sanchez, T., Narayan, T. C. \& Dinca, M. Postsynthetic tuning of hydrophilicity in pyrazolate MOFs to modulate water adsorption properties. Energy Environ. Sci. 6, 2172 (2013).

28. Rieth, A. J. \& Dinca, M. Controlled gas uptake in metal-organic Frameworks with record ammonia sorption. J. Am. Chem. Soc. 140, 3461-3466 (2018).

29. Kim, H. et al. Water harvesting from air with metal-organic frameworks powered by natural sunlight. Science 356, 430-434 (2017).

30. Rieth, A. J., Yang, S., Wang, E. N. \& Dinca, M. Record atmospheric fresh water capture and heat transfer with a material operating at the water uptake reversibility limit. ACS Cent. Sci. 3, 668-672 (2017).

31. Babin, V., Leforestier, C. \& Paesani, F. Development of a "first principles" water potential with flexible monomers: dimer potential energy surface, VRT spectrum, and second virial coefficient. J. Chem. Theory Comput. 9, 5395-5403 (2013).

32. Babin, V., Medders, G. R. \& Paesani, F. Development of a "first principles" water potential with flexible monomers. II: Trimer potential energy surface, third virial coefficient, and small clusters. J. Chem. Theory Comput. 10, 1599-1607 (2014).

33. Medders, G. R., Babin, V. \& Paesani, F. Development of a "first-principles" water potential with flexible monomers. III. Liquid phase properties. J. Chem. Theory Comput. 10, 2906-2910 (2014).

34. Medders, G. R. \& Paesani, F. Infrared and Raman spectroscopy of liquid water through "first-principles" many-body molecular dynamics. J. Chem. Theory Comput. 11, 1145-1154 (2015)

35. Reddy, S. K. et al. On the accuracy of the MB-pol many-body potential for water: Interaction energies, vibrational frequencies, and classical thermodynamic and dynamical properties from clusters to liquid water and ice. J. Chem. Phys. 145, 194504 (2016)

36. Rieth, A. J., Tulchinsky, Y. \& Dinca, M. High and reversible ammonia uptake in mesoporous azolate metal-organic frameworks with Open $\mathrm{Mn}, \mathrm{Co}$, and $\mathrm{Ni}$ sites. J. Am. Chem. Soc. 138, 9401-9404 (2016).

37. Tan, K. et al. Water reaction mechanism in metal-organic frameworks with coordinatively unsaturated metal ions: MOF-74. Chem. Mater. 26, 6886-6895 (2014).

38. Wright, A. M., Rieth, A. J., Yang, S., Wang, E. N. \& Dinca, M. Precise control of pore hydrophilicity enabled by post-synthetic cation exchange in metalorganic frameworks. Chem. Sci. 9, 3856 (2018).

39. Bernardina, S. D. et al. Water in carbon nanotubes: the peculiar hydrogen bond network revealed by infrared spectroscopy. J. Am. Chem. Soc. 138, 10437-10443 (2016).

40. Eaves, J. D. et al. Hydrogen bonds in liquid water are broken only fleetingly. Proc. Natl Acad. Sci. USA 102, 13019-13022 (2005).

41. Du, Q., Freysz, E. \& Shen, Y. R. Surface vibrational spectroscopic studies of hydrogen-bonding and hydrophobicity. Science 264, 826-828 (1994).

42. Sun, S. M. et al. Orientational distribution of free $\mathrm{OH}$ groups of interfacial water is exponential. Phys. Rev. Lett. 121, 246101 (2018).

43. Paesani, F. Getting the right answers for the right reasons: toward predictive molecular simulations of water with many-body potential energy functions. Acc. Chem. Res. 49, 1844-1851 (2016).

44. Medders, G. R. \& Paesani, F. Dissecting the molecular structure of the air/ water interface from quantum simulations of the sum-frequency generation spectrum. J. Am. Chem. Soc. 138, 3912-3919 (2016).

45. Medders, G. R. \& Paesani, F. Water dynamics in metal-organic frameworks: effects of heterogeneous confinement predicted by computational spectroscopy. J. Phys. Chem. Lett. 5, 2897-2902 (2014).

46. Terranova, Z. L., Agee, M. M. \& Paesani, F. Water structure and dynamics in Homochiral $\mathrm{Zn}(\mathrm{l}-\mathrm{L})(\mathrm{X})$ metal-organic frameworks. J. Phys. Chem. C. 119, 18239-18247 (2015).

47. Terranova, Z. L. \& Paesani, F. The effects of framework dynamics on the behavior of water adsorbed in the $\mathrm{Zn}(\mathrm{l}-\mathrm{L})(\mathrm{Cl})$ and Co-MOF-74 metal-organic frameworks. Phys. Chem. Chem. Phys. 18, 8196-8204 (2016).
48. Errington, J. R. \& Debenedetti, P. G. Relationship between structural order and the anomalies of liquid water. Nature 409, 318-321 (2001).

49. Rezus, Y. L. A. \& Bakker, H. J. On the orientational relaxation of HDO in liquid water. J. Chem. Phys. 123, 114502 (2005).

50. Krynicki, K., Green, C. D. \& Sawyer, D. W. Pressure and temperaturedependence of self-diffusion in water. Faraday Discuss. 66, 199 (1978)

51. Henderson, M. A. The interaction of water with solid surfaces: fundamental aspects revisited. Surf. Sci. Rep. 46, 1-308 (2002).

52. Kubelka, P. \& Munk, F. An article on optics of paint layers. Z. Tech. Phys. 12, 593 (1931).

53. Martinez, L., Andrade, R., Birgin, E. G. \& Martinez, J. M. PACKMOL: a package for building initial configurations for molecular dynamics simulations. J. Comput. Chem. 30, 2157-2164 (2009).

54. Smith, W. \& Forester, T. R. DL_POLY_2.0: a general-purpose parallel molecular dynamics simulation package. J. Mol. Graph. 14, 136-141 (1996).

55. Tuckerman, M. E. Statistical mechanics: Theory and molecular simulation (Oxford University Press, 2010).

56. Leach, A. R. Molecular modeling: Principles and applications (Pearson Prentice Hall, 2001).

\section{Acknowledgements}

Studies of small molecule interactions with metal nodes in MOFs are supported through a CAREER grant from the National Science Foundation to M.D. (DMR 1452612). A.J.R. is supported by the Martin Family Fellowship for Sustainability. A.J.R and M.D. thank the Abdul Latif Jameel World Water and Food Security Lab for seed funding for water capture. Theoretical research is supported by the Department of Energy through grant no. DE-SC0019333 to F.P. and used computational resources of the Extreme Science and Engineering Discovery Environment (XSEDE), which is supported by the National Science Foundation through grant no. ACI-1053575 under allocation TG-CHE110009, as well as of the Air Force Office of Scientific Research through grant no. FA9550-16-1-0327.

\section{Author contributions}

The paper was written through contributions of all authors. A.J.R. and K.M.H. contributed equally to this work. A.J.R. synthesized materials and designed and performed infrared experiments. K.M.H. designed and performed theoretical calculations. M.D. and F.P. designed and supervised research.

\section{Competing interests}

A.J.R. and M.D. are inventors on a patent pertaining to the materials discussed herein. K.M.H. and F.P. declare no competing interests.

\section{Additional information}

Supplementary information is available for this paper at https://doi.org/10.1038/s41467 019-12751-z.

Correspondence and requests for materials should be addressed to M.Dă. or F.P.

Reprints and permission information is available at http://www.nature.com/reprints

Peer Review Information Nature Communications thanks the anonymous reviewers for their contribution to the peer review of this work.

Publisher's note Springer Nature remains neutral with regard to jurisdictional claims in published maps and institutional affiliations.

Open Access This article is licensed under a Creative Commons Attribution 4.0 International License, which permits use, sharing, adaptation, distribution and reproduction in any medium or format, as long as you give appropriate credit to the original author(s) and the source, provide a link to the Creative Commons license, and indicate if changes were made. The images or other third party material in this article are included in the article's Creative Commons license, unless indicated otherwise in a credit line to the material. If material is not included in the article's Creative Commons license and your intended use is not permitted by statutory regulation or exceeds the permitted use, you will need to obtain permission directly from the copyright holder. To view a copy of this license, visit http://creativecommons.org/ licenses/by/4.0/

(C) The Author(s) 2019 